TRANSACTIONS OF THE

AMERICAN MATHEMATICAL SOCIETY

Volume 348, Number 5, May 1996

\title{
HYPERFINITE TRANSVERSAL THEORY. II
}

\author{
BOŠKO ŽIVALJEVIĆ
}

\begin{abstract}
We continue the investigation of validity of Hall's theorem in the case of the Loeb space $L(\mathcal{H})$ of an internal, uniformly distributed, hyperfinite measure space $\mathcal{H}=(\Omega, \mathcal{A}, \mu)$ initiated in1992 by the author. Some new classes of graphs are introduced for which the measure theoretic version of Hall's theorem still holds.
\end{abstract}

\section{INTRODUCTION}

This paper reports on the second part of the author's project devoted to the investigation of the validity of Hall's theorem in the setting of the Loeb space $L(\mathcal{H})$ of a uniformly distributed, internal measure space $\mathcal{A}=(\Omega, \mathcal{A}, \mu)$, initiated in [Ži1].

The motivating (and more general) question related to an arbitrary bounded measure space $M$ was originally raised by Nash-Williams in [NW]. He asked whether Hall's theorem still holds when the bipartite graph in question is taken to be a measurable subset of the product of an arbitrary nonatomic measure space $M$ with itself, and when the cardinality of a set is replaced by its measure. It is known that in general the answer to Nash-Williams' question is negative. Artstein ([Ar]) gives a simple example of a compact subset of the unit square $[0,1] \times[0,1]$ which satisfies Hall's condition with respect to Lebesgue measure $m$ in $[0,1]$ and which, in turn, does not possess a measurable matching (in our terminology). The construction of the example [Ži1, p. 396], carried out in the setting of the unit cube $[0,1] \times[0,1]$, gives another such example. In the positive direction Artstein proves that if a closed subset $\Gamma$ of the product of two Polish (i.e., complete separable metric) spaces $G$ and $B$ satisfies Hall's condition, and all of its vertical sections are compact, then $\Gamma$ possesses a so-called mixed matching: given two probability measures $\sigma$ and $\rho$ in $G$ and $B$ respectively, a mixed matching $\mu$ of a subset $\Gamma$ of $G \times B$ is a measurable function $\mu$ assigning to every $g \in G$ a probability measure $\mu(g)$ in $B$ so that the support of $\mu(g)$ is included in $\Gamma(g)$ (the vertical section of $\Gamma$ at $g$ ) and such that $\mu$ preserves the density, i.e., $\rho(A)=\int_{G} \mu(g)(A) d \sigma$ for all closed $A \subseteq B$. Mauldin further shows in $[\mathrm{Ma}]$ that any Lebesgue measurable subset $\Gamma$ of $[0,1] \times[0,1]$ all of whose vertical and horizontal sections are of positive Lebesgue measure possesses a one-to-one Borel uniformization, i.e., there exists a Borel measurable, one-toone function $f$, whose graph is a subset of $\Gamma$, and whose domain and range have Lebesgue measure one.

Received by the editors August 7, 1994 and, in revised form, June 5, 1995.

1991 Mathematics Subject Classification. Primary 03H04, 03E15; Secondary 04A15, 05C99, 28E05, 54H05.

Key words and phrases. Nonstandard measure theory, transversal theory, Hall's theorem, descriptive set theory of internal sets. 
However the most interesting example of a nontrivial measure space $M$ in which the measure theoretic analogue of Hall's theorem holds in the way envisaged by Nash-Williams is undoubtedly the Loeb space $L(\mathcal{H})$ of a uniformly distributed, hyperfinite measure space $\mathcal{H}=(\Omega, \mathcal{A}, \mu)$ arising in Nonstandard Measure Theory. $L(\mathcal{H})$ consists of an infinite hyperfinite set $\Omega$ of cardinality $K$ and the Loeb measure $L(\mu)$ of an internal counting measure $\mu$ defined as $\mu(A)=|A| / H$ for every $A \in \mathcal{A}$, $\mathcal{A}$ being the internal power set of $\Omega$. Here $H$ is an infinite integer for which we require, for purely technical reasons, that $H / K \approx 0$, making $\mathcal{H}$ an unbounded Loeb space. The setting in which we formulate the problem is the following. We take two Loeb measurable subsets $X$ and $Y$ of $\Omega$ of finite Loeb measure and a subset (here called a graph) $\Gamma$ of the product $X \times Y$ of different set theoretical "complexity". We let $\Gamma$ satisfy Hall's condition and ask if the full or the approximation form of Hall's Theorem is true (for detailed definitions see $\S 0$ ). One has to stress here that Hall's condition is necessary for the existence of Borel matchings. By a result of Henson and Ross (see [HeRo]), any injective Borel function, in the setting of the Descriptive Set Theory of Internal Sets, necessarily preserves any given counting Loeb measure. Therefore, if a graph possesses a Borel matching saturating almost all of $X$, then it must satisfy Hall's condition.

The types of graphs that have been considered so far in [Ži1] are the so-called $\Pi_{1}^{0}(\kappa)$ graphs, i.e., the intersections of $\kappa$ many internal graphs, and $\Sigma_{1}^{0}(\kappa)$ monotone graphs, i.e., the monotone unions of $\kappa$ many internal graphs. While it was shown that Hall's theorem still holds true for $\Pi_{1}^{0}(\kappa)$ graphs, yielding the hyperfinite version of Transversal Theory, it does not hold for an arbitrary $\Sigma_{1}^{0}(\kappa)$ monotone graph. Rather, every $\Sigma_{1}^{0}(\kappa)$ monotone graph satisfying Hall's condition possesses internal partial matchings (the function $\mathcal{M}$ in Hall's theorem) of arbitrary small measure defects (again, for definitions see §0). Also, an example in [Ži1] showed that this result is the best possible, i.e., there exists a $\Sigma_{1}^{0}$ graph satisfying Hall's condition which, nevertheless, does not possess a measure preserving matchings saturating almost all of $X$.

The purpose of this paper is to present some more interesting classes of graphs for which Hall's theorem still holds true.

In $\S 0$ we repeat some necessary definitions and notation introduced in [Ži1]. The reader familiar with [Ži1] might skip this section.

In $\S 1$ we consider Loeb measurable graphs in which "height" and "width" are suitably bounded. Here the "height" ("width") of a graph $\Gamma$ is defined to be a function $d_{\Gamma}$ assigning to every $x$ in $X(Y)$ the measure of the $Y(X)$ section of $\Gamma$ at $x$. We prove, among other results, that the approximation version of Hall's theorem holds true for $r$ regular graphs $(r>0)$, and that the full version of Hall's theorem holds true for graphs which are of full measure in some finite union of measurable rectangles. These results extend (and use) the corresponding results from [Ži1]. Keisler's Fubini theorem and the Loeb measure variant of a result of Bollobas and Varopoulos ([BoVa] and [Ži2]) have been used in the proof.

In $\S 2$ we investigate Souslin graphs. (A graph is Souslin if it can be obtained by applying Souslin operation to a family of internal graphs.) We define a certain set function $J$ and prove, by using Choquet's Capacitability Theorem, that if $J$ is strongly subadditive on an internal algebra $\mathcal{K}$ of graphs then any Souslin over $\mathcal{K}$ graph $\Gamma$ satisfying Hall's condition possesses internal matchings of arbitrary small measure defects. At the end, using the idea of the proof of Choquet's Capacitability 
Theorem, we give an interesting sufficient condition in terms of the Souslin representation of $\Gamma$ insuring the validity of the approximation version of Hall's Theorem.

In $\S 3$ two new classes of graphs are introduced: $\Pi_{2}^{0}(\kappa)$ graphs of depth $\omega$ and $\kappa$-Souslin graphs. Sufficient conditions for the approximation version of Hall's Theorem for those types of graphs are given. A necessary and sufficient condition for an arbitrary $\Pi_{2}^{0}$ graph insuring the validity of the approximation form of Hall's theorem is also proved. All the above conditions are expressed in terms of a specific set representation of the graph $\Gamma$.

The most satisfactory version of Hall's Theorem is presented in $\S 4$. A lifting of a graph $\Gamma$ is introduced as a graph $\Delta$ for which $\Delta(x)=\Gamma(x)$ for almost all $x$. It was shown that if $\Gamma$ satisfies Hall's condition then its lifting $\Delta$ must also satisfy Hall's condition. This in turn transforms the question of the validity of Hall's theorem to the questions of when a particular graph allows an internal, $\Sigma_{1}^{0}$ or $\Pi_{1}^{0}$ lifting. In a series of papers [SchŽi], [Ži3], [Ži4] and [Ži5], the structure of graphs, all of whose vertical sections are of fixed low level Borel class, was explored and the existence of their liftings proved. The results of those papers are used in this section. The broadest class of graphs for which the full version of Hall's theorem holds is the class of $\Sigma_{1}^{1}$ graphs with $\Pi_{1}^{0}, Y$ sections and the broadest class of graphs for which the approximation version of Hall's theorem holds is the class of $\Pi_{1}^{1}$ graphs with $\Sigma_{1}^{0}, Y$ sections.

Finally, in $\S 5$ a new measure $L(\nu)$ is introduced in the product $\Omega \times \Omega$. It was shown that all graphs of finite $L(\nu)$ measure satisfying Hall's condition possess almost surely an internal matching saturating almost all of the domain of the graph.

Some of the results of this paper are based on my doctoral dissertation which I defended at the Department of Mathematics of the University of Illinois at UrbanaChampaign. I would like to thank once more my adviser Professor Ward Henson for help he offered during the research on my dissertation and for his support and encouragement during these past six years after my graduation.

\section{Definitions and notation}

In this section we shall briefly overview the major definitions and notation. For more detailed introduction the reader is referred to [Ži1] and references there. For an introduction to Nonstandard Analysis the reader is referred to the book of A. Hurd and P. Loeb ([HuLo]) and for the treatment of Descriptive Set Theory of Hyperfinite Sets to [KKLM].

An unbounded, hyperfinite, uniformly distributed counting space $\mathcal{H}$ is a triple $(\Omega, \mathcal{A}, \mu)$ where $\Omega$ is a hyperfinite set, $\mathcal{A}$ the internal algebra of all internal subsets of $\Omega$, and $\mu$ an internal counting measure on $\mathcal{A}$ defined as $\mu(A)=|A| / H, H$ being a fixed hyperfinite integer. The word "unbounded" reflects the fact that $\mu(\Omega)$ is an infinite real number. The associated Loeb space $L(\mathcal{H})$ is a standard measure space $(\Omega, L(\mathcal{A}), L(\mu))$, with $L(\mathcal{A})$ being a standard $\sigma$ algebra of Loeb measurable subsets of $\Omega$ and $L(\mu)$ a standard $\sigma$ additive measure defined on $L(\mathcal{A})$. The space $\Omega$ is an environment from which the sets of vertices of finite Loeb measure are picked up. $\Omega$ is chosen to be of infinite measure from purely technical reasons and we shall almost exclusively be interested in Loeb measurable sets of finite measure. Here a set $M$ of infinite outer measure is said to be Loeb measurable if $M \cap B$ is Loeb measurable for every set $B$ of finite Loeb measure.

The Borel hierarchy of subsets of an internal set $X$ is defined as usual. Internal 
subsets of $X$ are called $\Pi_{0}^{0}$ or $\Sigma_{0}^{0}$ sets. If we have defined the classes of $\Pi_{\beta}^{0}$ and $\Sigma_{\beta}^{0}$ sets for every $\beta<\alpha$, then we say that a set belongs to the class $\Pi_{\alpha}^{0}\left(\Sigma_{\alpha}^{0}\right)$ if it is an intersection (union) of a countable sequence of sets each member of which belongs to a class $\Pi_{\beta}^{0}$ or $\Sigma_{\beta}^{0}$ for some $\beta<\alpha$. A set is Borel if it is $\Pi_{\alpha}^{0}$ or $\Sigma_{\alpha}^{0}$ for some $\alpha<\omega_{1}$.

A set $S$ is $\Sigma_{1}^{1}$ if it is the projection of a Borel subset of the product of two internal sets. It is well known (Henson) that a set $S$ is $\Sigma_{1}^{1}$ if and only if it is Souslin, i.e., it can be represented as $\bigcup_{\xi \in \omega^{\omega}} \bigcap_{n \in \omega} A_{\xi \mid n}$ for some sequence $A_{s},\left(s \in \omega^{<\omega}\right)$ of internal sets. If the sets $A_{s}$ are members of some algebra $\mathcal{K}$ then $S$ is called Souslin over $\mathcal{K}$. Reader is referred to two standard references [He] and [KKLM] for the treatment of Descriptive Set Theory of Internal Sets.

Given two Loeb measurable sets of finite measure $X$ and $Y$, a graph in $X \times Y$ is an arbitrary subset $\Gamma$ of the product $X \times Y$. For $A \subseteq X$ we define the image of $A$ under $\Gamma, \Gamma(A)$, as $\Gamma(A)=\{y:(\exists x \in A)(x, y) \in \Gamma\}$. When $A$ is a singleton $\{x\}$ we simply write $\Gamma(x)$ instead of $\Gamma(\{x\})$ and call it the $Y$ section of $\Gamma$ at $x$.

The inverse graph $\Gamma^{-1}$ the domain $\operatorname{dom}(\Gamma)$ and the range $\operatorname{ran}(\Gamma)$ are defined as usual.

A partial matching $\mathcal{M}$ of $\Gamma$ is an injective function whose graph is a subset of $\Gamma$. We say that $x$ is saturated by $\mathcal{M}$ if $x \in \operatorname{dom}(\mathcal{M})$.

In almost all the situations that follow, the domain of $\mathcal{M}$ will be measurable. In that case the measure defect of $\mathcal{M}$ is defined as $L(\mu)(X)-L(\mu)(\operatorname{dom}(\mathcal{M}))$, i.e., the measure of the set of vertices in $X$ unsaturated by $\mathcal{M}$. We shall usually omit referring to the set $X$ while talking about the measure defect of the graph $\Gamma$ if there is no danger of confusion.

Given a graph $\Gamma \subseteq X \times Y$, we can formulate three versions of Hall's condition for $\Gamma$ depending on whether $\Gamma$ maps internal sets into Loeb measurable sets or not. In the first case we say that $\Gamma$ satisfies Hall's condition if and only if

$$
L(\mu)(\Gamma(A)) \geq L(\mu)(A)
$$

for every internal $A \subseteq X$. In the second we say that $\Gamma$ satisfies the inner Hall's condition if

$$
L(\mu)_{i}(\Gamma(A)) \geq L(\mu)(A)
$$

for every internal $A \subseteq X$, and the outer Hall's condition if

$$
L(\mu)_{e}(\Gamma(A)) \geq L(\mu)(A)
$$

for every internal $A \subseteq X$. Here $L(\mu)_{i}$ and $L(\mu)_{e}$ are the inner and the outer Loeb measures associated with $\mu$.

We are interested in discovering those graphs $\Gamma$ for which one of the above conditions is equivalent to the existence of a Borel $\left(\Sigma_{1}^{1}\right)$ matching saturating almost all of $X$ or a Borel $\left(\Sigma_{1}^{1}\right)$ partial matching of the prescribed measure defect. One should point out here that it is enough to look for $\Sigma_{1}^{0}$ (partial) matchings. It follows from a result of Henson and Ross (see [HeRo]) that given a $\Sigma_{1}^{1}$ function $f$ and a bounded Loeb measure $L(\mu), f$ is $L(\mu)$ a.e. equal to a $\Sigma_{1}^{0}$ function $g$, the graph of which is a subset of $f$. The same is also true for every $\Pi_{1}^{1}$ function $f$ ([Ži5]). In fact, one can show that given a bounded Loeb measure $L(\mu)$ in $X$ and a function $f$ whose graph is a universally Loeb measurable subset of $X \times Y(e . g$. , a member of the list 
$\sigma$ algebra generated by $\Sigma_{1}^{1}$ sets) and at the same time countably determined, then $f$ is $L(\mu)$ a.e. equal to a $\Sigma_{1}^{0}$ sub-function $g$. Thus, we can restrict our attention to $\Sigma_{1}^{0}$ matchings, only.

We say that the full form of Hall's theorem holds for $\Gamma$ if the Hall's condition is equivalent to the existence of a $\Sigma_{1}^{0}$ matching of $\Gamma$ saturating almost all of $X$. The approximation version of Hall's theorem holds for $\Gamma$ if Hall's condition is equivalent to the existence of internal matchings of $\Gamma$ of arbitrary small measure defects.

Throughout this paper we assume that our nonstandard universe is at least $\aleph_{1}$ saturated.

\section{Graphs of positive Loeb measure}

In this section we are interested in Loeb measurable graphs $\Gamma$ of positive $L(\mu \times \mu)$ measure in the product of $X \times Y$. Here, $\mu \times \mu$ is the internal product of the internal measure $\mu$ with itself and $L(\mu \times \mu)$ is the associated Loeb measure. The degree $d_{\Gamma}$ of $\Gamma \subseteq X \times Y$ is defined as

$$
\begin{aligned}
& d_{\Gamma}(x)=L(\mu)(\Gamma(x)) \quad \text { for } \quad x \in X, \\
& d_{\Gamma}(y)=L(\mu)\left(\Gamma^{-1}(y)\right) \quad \text { for } \quad y \in Y .
\end{aligned}
$$

The fact that $\Gamma$ is of positive measure in $X \times Y$ will insure that the degree function of $\Gamma$ is not identically equal to zero. If we succeed in bounding the degree function from above and from below in a certain way, then, depending on how good the bounds are, $\Gamma$ will possess matchings of certain measure defects. For example, if $\Gamma$ is a regular graph (i.e., the degree function is constant and positive) then we shall prove that $\Gamma$ possesses internal matchings of arbitrary small measure defects. Similarly, if $\Gamma$ is a finite union of measurable rectangles then $\Gamma$ must possess a Borel matching saturating almost all of $X$.

We start with the proposition which extends Proposition 10.5 in [Ži1]. Approximating the graph $\Gamma$ from within by a $\Sigma_{1}^{0}$ graph and using Keisler's Fubini theorem we extend Proposition 10.5 ([Ži1]) from $\Sigma_{1}^{0}(\kappa)$ monotone graphs to $L(\mu \times \mu)$ measurable graphs. Recall that, assuming $\kappa^{+}$saturation, $\Sigma_{1}^{0}(\kappa)$ monotone graphs are always universally Loeb measurable, i.e., measurable with respect to any Loeb measure.

Proposition 1.1. Let $X$ and $Y$ be Loeb measurable sets of finite measure. Let $\Gamma \subseteq X \times Y$ be a $L(\mu \times \mu)$ measurable graph. Suppose that

$$
d_{\Gamma}(\beta) \leq r_{1} \text { a.e. on } Y \text { and } d_{\Gamma}(\alpha) \geq r_{2} \text { a.e. on } X \text {. }
$$

Then, for every $\epsilon>0, X$ can be partitioned into $\left\lceil r_{1} / r_{2}\right\rceil$ Loeb measurable parts such that there exists an internal function $\mathcal{M}$ in $\Gamma$, the restriction to each member of the partition of which is injective and such that $L(\mu)(X \backslash \operatorname{dom}(\mathcal{M})) \leq \epsilon$.

Proof. Let $\Gamma_{n} \subseteq \Gamma$ be an internal graph with $L(\mu)\left(\Gamma \backslash \Gamma_{n}\right) \leq 1 / n$. The graph $\Delta=$ $\bigcup_{n \in \omega} \Gamma_{n}$ is a $\Sigma_{1}^{0}$ graph. By Keisler's Fubini theorem (see [StBa]), $d_{\Delta}(y)=d_{\Gamma}(y)$ for almost all $y \in Y$, and $d_{\Delta}(x)=d_{\Gamma}(x)$ for almost all $x \in X$, where $d_{\Delta}$ and $d_{\Gamma}$ are the corresponding degree functions of the graphs $\Gamma$ and $\Delta$. Therefore, we have $d_{\Delta}(y) \leq r_{1}$ and $d_{\Delta}(x) \geq r_{2}$ for almost all $y \in Y$ and almost all $x \in X$. The result now follows from Proposition 10.5 in [Ži1] applied to the graph $\Delta \subseteq \Gamma$. 
Let $f$ be a real valued Loeb measurable function defined on a set of finite measure. The essential infimum $\mathfrak{i}(f)$ and the essential supremum $\mathfrak{s}(f)$ of $f$ are defined as $\mathfrak{i}(f)=\sup \left\{r: L(\mu)\left(f^{-1}(]-\infty, r[)\right)=0\right\}$ and $\mathfrak{s}(f)=\inf \left\{r: L(\mu)\left(f^{-1}(] r,+\infty[)\right)=\right.$ $0\}$.

Corollary 1.2. Let $X$ and $Y$ be Loeb measurable sets of finite measure. Let $\Gamma \subseteq$ $X \times Y$ be a $L(\mu \times \mu)$ measurable graph and let $d_{\Gamma} \mid X\left(d_{\Gamma} \mid Y\right)$ be the restriction of the degree function $d_{\Gamma}$ to $X(Y)$. Suppose that $\mathfrak{s}\left(d_{\Gamma} \mid Y\right) \leq \mathfrak{i}\left(d_{\Gamma} \mid X\right)$. Then, for every $\epsilon>0, \Gamma$ has an internal matching of measure defect $\leq \epsilon$.

Proof. Let $r_{1}=\mathfrak{s}\left(d_{\Gamma} \mid Y\right)$ and $r_{2}=\mathfrak{i}\left(d_{\Gamma} \mid X\right)$. Then, $\left\lceil r_{1} / r_{2}\right\rceil=1$.

A special case of the above corollary is when $\Gamma$ is $r$-regular for some positive $r$.

Corollary 1.3. Let $X$ and $Y$ be Loeb measurable sets of finite measure and let $\Gamma \subseteq X \times Y$ be a Loeb measurable graph. Suppose that $\Gamma$ is $r$-regular for some $r>0$, i.e., $d_{\Gamma}(x)=r$ a.e on $X \cup Y$. Then for every $\epsilon>0, \Gamma$ has an internal matching of measure defect $\leq \epsilon$.

A similar example to the one given at the end of [Ži1] shows that the above results are the best possible. Although an $r$ regular measurable graph $\Gamma$ possesses internal matchings of arbitrary small measure defects it is not true that $\Gamma$ would necessarily possess a Borel matching saturating almost all of $X$.

Example 1.4. Let $T=\left\{1, \ldots, 2^{H}\right\}$, with $H$ an infinite natural number. Let $\mu$ be any internal, uniformly distributed, counting measure such that $\mu(T)$ is finite. Let $\Gamma_{n}$ be the set of points in $T \times T$ contained inside the triangle with vertices $\left(2^{H-n}, 1\right),\left(2^{H}, 1\right)$ and $\left(2^{H}, 2^{H-n}\right), n \geq 1$ being a standard integer. $\Gamma=\bigcup_{n \in \omega} \Gamma_{n}$ is a Loeb measurable 1/2-regular graph. In the same manner as in the example on the page 396 of [Ži1] one can show that $\Gamma$ does not possess a measure preserving matching saturating almost all of $T$. In particular, $\Gamma$ does not possess a Borel or a $\Sigma_{1}^{1}$ matching saturating almost all of $T$.

In contrast to the above counterexample, the Loeb measurable graphs of full measure in the product $X \times Y$ possess Borel matchings saturating almost all of $X$ provided that $L(\mu)(Y) \geq L(\mu)(X)$.

Proposition 1.5. Let $X$ and $Y$ be Loeb measurable sets of finite measure with $L(\mu)(X) \leq L(\mu)(Y)$. Let $\Gamma \subseteq X \times Y$ be a Loeb measurable graph of full measure in $X \times Y$, i.e., $L(\mu \times \mu)(\Gamma)=L(\mu)(X) \cdot L(\mu)(Y)$. Then, there exist a $\Sigma_{1}^{0}$ matching of $\Gamma$ saturating almost all of $X$.

Proof. By Corollary 1.2., there exists an internal matching $\mathcal{M}_{1}$ of $\Gamma$ such that

$$
L(\mu)(X)-L(\mu)\left(\operatorname{dom}\left(\mathcal{M}_{1}\right)\right) \leq \frac{1}{2} \cdot L(\mu)(X)
$$

The graph

$$
\Gamma_{1}=\Gamma \cap\left(X \backslash \operatorname{dom}\left(\mathcal{M}_{1}\right)\right) \times\left(Y \backslash \operatorname{ran}\left(\mathcal{M}_{1}\right)\right)
$$

is of full measure in $\left(X \backslash \operatorname{dom}\left(\mathcal{M}_{1}\right)\right) \times\left(Y \backslash \operatorname{ran}\left(\mathcal{M}_{1}\right)\right)$. Moreover, following the fact that $L(\mu)\left(\operatorname{dom}\left(\mathcal{M}_{1}\right)\right)=L(\mu)\left(\operatorname{ran}\left(\mathcal{M}_{1}\right)\right)$, we have

$$
L(\mu)\left(X \backslash \operatorname{dom}\left(\mathcal{M}_{1}\right)\right) \geq L(\mu)\left(Y \backslash \operatorname{ran}\left(\mathcal{M}_{1}\right)\right) .
$$


Applying Corollary 1.2. to $\Gamma_{1}$ again we obtain an internal matching $\mathcal{M}_{2}$ of $\Gamma_{1}$ such that

$$
L(\mu)(X)-L(\mu)\left(\operatorname{dom}\left(\mathcal{M}_{1}\right)\right)-L(\mu)\left(\operatorname{dom}\left(\mathcal{M}_{2}\right)\right) \leq \frac{1}{2^{2}} \cdot L(\mu)(X) .
$$

It is clear that $\mathcal{M}_{1} \cup \mathcal{M}_{2}$ is an injective function. Continuing inductively we obtain a sequence $\mathcal{M}_{n},(n \in \omega)$ of internal matchings of $\Gamma$ such that

$$
L(\mu)(X)-\sum_{i=1}^{n} L(\mu)\left(\mathcal{M}_{i}\right) \leq \frac{1}{2^{n}} .
$$

The union $\bigcup_{n \in \omega} \mathcal{M}_{k}$ is the required $\Sigma_{1}^{0}$ matching.

If we assume $\kappa$ saturation for an infinite cardinal $\kappa$ then, in fact, the graph $\Gamma$ above possesses $\kappa$ many disjoint $\Sigma_{1}^{0}$ matchings. This follows from the fact that the union of less than $\kappa$ many internal sets of measure zero is also of measure zero.

Example 1.6. It is not true that a graph of full measure in the product $X \times Y$ possesses an internal matching saturating almost all of the domain of the graph. In other words, $\Sigma_{1}^{0}$ above cannot be replaced by $\Sigma_{0}^{0}$. Let $T=\left\{1, \ldots, 2^{H}\right\}$, where $H$ is an infinite integer. Let $P_{n}$ be a rectangle defined as

$$
\left.\left.P_{n}=\right] 2^{H-n-1}, 2^{H-n}\right] \times T,
$$

and let $\Gamma$ be defined as $\Gamma=\bigcup_{n \in \omega} P_{n}$. Then, $\Gamma$ is a $\Sigma_{1}^{0}$ graph of full measure in $T \times T$. However, any internal matching in $\Gamma$ is covered by finitely many sets $P_{n}$ and thus, cannot have the domain of full measure in $\mathrm{T}$.

The next proposition uses a result of Bollobas and Varopoulos (see [BoVa]) applied to the Loeb spaces. The same result is proved as a corollary to a version of Rado's theorem (Theorem I) in [Ži2]. The corollary in question (page 206 in [Ži2]) states that if a sequence $M_{i},(i \in \Lambda)$ of Loeb measurable sets of finite measure and a sequence $a_{i},(i \in \Lambda)$ of real numbers satisfy $L(\mu)\left(\bigcup_{i \in e}\left(M_{i}\right)\right) \geq \sum_{i \in e} a_{i}$ for every finite $e \subseteq \Lambda$ then there exist Loeb measurable sets $N_{i} \subseteq M_{i}$ such that $L(\mu)\left(N_{i} \cap N_{j}\right)=0$ for $i \neq j$ and $L(\mu)\left(N_{i}\right)=a_{i}$.

Proposition 1.7. Let $X$ and $Y$ be Loeb measurable sets of finite measure. Let $\Gamma \subseteq X \times Y$ be a graph such that

$$
\Gamma \subseteq \bigcup_{n \in \omega} M_{n} \times N_{n}
$$

where the sets $M_{n}$ and $N_{n}(n \in \omega)$ are Loeb measurable with $M_{n} \cap M_{m}=\emptyset$, $(m \neq n)$, and such that $\Gamma$ is of full measure in $\bigcup_{n \in \omega} M_{n} \times N_{n}$. Suppose that Hall's condition is satisfied. Then, $\Gamma$ possesses a $\Sigma_{1}^{0}$ matching saturating almost all of $X$.

Proof. Let $L(\mu)\left(M_{n}\right)=a_{n}$. Hall's condition implies that for every finite $e \subseteq \omega$ we have

$$
L(\mu)\left(\bigcup_{n \in e} N_{n}\right) \geq \sum_{n \in \omega} a_{n} .
$$

By the result of Bollobas and Varopoulos there exists a family $H_{n},(n \in \omega)$ of Loeb measurable sets satisfying $H_{n} \subseteq N_{n}$ and $L(\mu)\left(H_{n}\right)=a_{n}$. Now, the fact that $\Gamma$ is 
of full measure in every $M_{n} \times H_{n}$ implies that $\Gamma$ possesses a $\Sigma_{1}^{0}$ matching in every $M_{n} \times H_{n}$ saturating almost all $M_{n}$. The union of such obtained $\Sigma_{1}^{0}$ matchings is again a $\Sigma_{1}^{0}$ matching saturating almost all $X$.

Remark 1.8. The above proposition does not hold if the requirement that the sets $M_{n}$ are mutually disjoint is released. Indeed, one can easily see that the graph in Example 1.4 can be obtained as a countable union of rectangles. On the other hand, if the sets $M_{n}$ are not mutually disjoint then $\Gamma$ possesses internal matchings of arbitrary small measure defects (by approximating each of the rectangles from within by internal rectangles). If the union $\bigcup_{n \in \omega} M_{n} \times N_{n}$ is reduced to a finite union of rectangles, then $\Gamma$ possesses a $\Sigma_{1}^{0}$ matching of measure defect 0 .

\section{SOUSLIN GRAPHS}

Recall that a graph $\Gamma \subseteq X \times Y$ is called Souslin (or $\Sigma_{1}^{1}$ ) if it is obtained by applying the Souslin operation on a family of internal graphs in the product $X \times Y$. If the internal graphs in question are members of an algebra of internal sets $\mathcal{K}$ then we call $\Gamma$ Souslin over $\mathcal{K}$. In this section we show that if the function $L(\mu)(X)-\delta(\Gamma)$, $\delta(\Gamma)$ being the measure defect of $\Gamma$, restricted to an algebra $\mathcal{K}$ of internal graphs is strongly subadditive then every Souslin over $\mathcal{K}$ graph satisfying Hall's condition possesses matchings of arbitrary small measure defects. Given $\Gamma \subseteq X \times Y$, with $X$ and $Y$ being of finite Loeb measure as usual, we call the measure defect of $\Gamma$ the number

$$
\begin{aligned}
& \delta(\Gamma)=\inf \{L(\mu)(A): A \subseteq X \text { internal, } \Gamma \text { has an internal matching } \\
& \text { saturating almost all of } X \backslash A\} \text {. }
\end{aligned}
$$

Notice that the measure defect of $\Gamma$ depends not only on the graph $\Gamma$ but on the set $X$ as well. In addition, internal matchings in the above definition could be substituted by Borel or Souslin matchings without changing the value of the defect. Saying that $\Gamma$ possesses internal matching of arbitrary small measure defects is equivalent to saying that $\delta(\Gamma)=0$.

The content of the following proposition has been proved in [Ži1], Proposition 2.2, 2.4 and Corollary 10.3.

Proposition 2.1. Let $X$ and $Y$ be Loeb measurable sets of finite measure. Let $\Gamma \subseteq X \times Y$ be $a \Pi_{1}^{0}$ or a $\Sigma_{1}^{0}$ graph. Then,

$$
\delta(\Gamma)=\sup \{L(\mu)(A)-L(\mu)(\Gamma(A)): A \subseteq X \text { internal }\} .
$$

If $\Gamma$ is $\Pi_{1}^{0}$, then there always exists an internal matching of $\Gamma$ of defect $\delta(\Gamma)$.

Let $X$ and $Y$ be as usual: Loeb measurable sets of finite measure. For any graph $\Gamma \subseteq X \times Y$ we define

$$
I(\Gamma)=L(\mu)(X)-\sup \{L(\mu)(A)-L(\mu)(\Gamma(A)): A \subseteq X, A \text { internal }\}
$$

provided that $\Gamma$ maps internal sets into measurable sets. While the measure defect $\delta(\Gamma)$ of $\Gamma$ is expressed in terms of internal matchings of $\Gamma$, the function $I$ is defined by using Hall's condition-like expression. In a certain sense, the function $I$ estimates the measures of the domains of maximal possible internal matchings of $\Gamma$ suggested 
by Hall's condition, whereas the measure defect $\delta(\Gamma)$ of $\Gamma$ actually gives that number explicitly. In such a way showing that the approximation version of Hall's theorem holds for $\Gamma$ is the same as proving that $I(\Gamma)=X$ implies $\delta(\Gamma)=0$. When $\Gamma$ is a $\Pi_{1}^{0}$ or $\Sigma_{1}^{0}$ graph, for example, we have $I(\Gamma)=L(\mu)(X)-\delta(\Gamma)$ and the above equivalence is true.

It is easy to prove using $\aleph_{1}$ saturation that $I$ passes trough countable, monotone unions and intersections of internal graphs, i.e., for a monotone sequence of internal graphs $\Gamma_{n},(n \in \omega)$ we always have $I\left(\bigcup_{n \in \omega} \Gamma_{n}\right)=\sup \left\{I\left(\Gamma_{n}\right): n \in \omega\right\}$ and $I\left(\bigcap_{n \in \omega} \Gamma_{n}\right)=\inf \left\{I\left(\Gamma_{n}\right): n \in \omega\right\}$. Also, $I$ is always positive and monotone function, i.e., $I(\Gamma) \geq I(\Delta) \geq 0$ for $\Gamma \supseteq \Delta$.

Let $\mathcal{K}$ be a fixed internal family of internal graphs in $X \times Y$ closed w.r.t. finite intersections and unions. We refer to the elements of $\mathcal{K}$ as simple graphs. Let $J$ be a new set function defined on $\mathcal{K}$ as $J(\Delta)=I(\Delta)$. Thus, $J(\Delta)=L(\mu)(X)-\delta(\Delta)$ for $\Delta \in \mathcal{K}$. Suppose that $J$ is strongly subadditive on $\mathcal{K}$, i.e.,

$$
J(\Gamma \cup \Delta)+J(\Gamma \cap \Delta) \leq J(\Gamma)+J(\Delta), \quad \Gamma, \Delta \in \mathcal{K} .
$$

Let $\mathcal{K}_{\sigma}$ be the family obtained by closing $\mathcal{K}$ w.r.t. countable unions. Following [Me] we can extend $J$ to the class $\mathcal{K}_{\sigma}$ by defining $J(G)=\sup \{J(\Gamma): \Gamma \in \mathcal{K}, \Gamma \subseteq G\}$. For any graph $G$ we define

$$
J(G)=\inf \left\{J(S): S \in \mathcal{K}_{\sigma}, S \supseteq G\right\} .
$$

The extended function $J$ is a $\mathcal{K}$-capacity, i.e., $J\left(\bigcup_{n \in \omega} M_{n}\right)=\sup \left(J\left(M_{n}\right)\right)$ for every increasing sequence $M_{n}(n \in N)$ of arbitrary sets and $J\left(\bigcap_{n \in \omega} A_{n}\right)=\inf \left(J\left(A_{n}\right)\right)$ for every decreasing sequence of elements of $\mathcal{K}$. The proof is essentially the same as one given in $[\mathrm{Be}]$, where it was shown that an internal, strongly subadditive, positive and monotone set function can be extended to a standard capacity defined on all subsets of a given internal set. The same result is also independently discovered by D. Ross ([Ro], Theorem 2.6) where a complete treatment of nonstandard capacities can be found. For the sake of completeness, however, we shall briefly sketch the proof of Ross and Bartossi's result here. For the details see [Be], [Ro] or [HeRo].

Proposition 2.2. The function $J$ defined on all the subsets of $X$ is a $\mathcal{K}$-capacity.

Proof. For every increasing sequence $K_{n},(n \in \omega)$ of elements of $\mathcal{K}$, with $K=$ $\bigcup_{n \in \omega} K_{n} \in \mathcal{K}$ we have $J(K)=\sup _{n \in \omega} J\left(K_{n}\right)$ (this follows from the fact that $\mathcal{K}$ is internal and $\aleph_{1}$-saturation). For every decreasing sequence $K_{n},(n \in \omega)$ of elements of $\mathcal{K}$, we have $J\left(\bigcap_{n \in \omega} K_{n}\right)=\inf \left(J\left(K_{n}\right)\right)$ (again an easy $\aleph_{1}$-saturation argument). Therefore, the conditions sufficient for the extension of a strongly subadditive, positive and monotone set function to a capacity (in a way done above) are met (see $[\mathrm{Me}]$, Theorem 23 ). The function $J$ is indeed a $\mathcal{K}$-capacity.

A set is called $\mathcal{K}$-capacitable if it can be approximated from within by elements of the set $\mathcal{K}_{\delta}$, where $\mathcal{K}_{\delta}$ is obtained by closing $\mathcal{K}$ w.r.t. countable intersections. In other words $S$ is capacitable if $J(S)=\sup \left\{J(P): P \subseteq S, P \in \mathcal{K}_{\delta}\right\}$. Souslin sets (over $\mathcal{K}$ ) are always capacitable w.r.t. any capacity.

Proposition 2.3. Let $X$ and $Y$ be Loeb measurable sets of finite measure. Let $\Gamma$ be a Souslin graph over $\mathcal{K}$ satisfying Hall's condition. Then, for every $\epsilon>0, \Gamma$ possesses an internal matching of measure defect $\leq \epsilon$. 
Proof. As $\Gamma$ is Souslin and $J$ a $\mathcal{K}$-capacity, $\Gamma$ is $J$-capacitable. That means that $\Gamma$ can be approximated from within by $\mathcal{K}_{\delta}$ sets, i.e.,

$$
J(\Gamma)=\sup \left\{J(P): P \subseteq \Gamma, P \in \mathcal{K}_{\delta}\right\} .
$$

On the other side, by the definition of $J(\Gamma)$, we have

$$
J(\Gamma)=\inf \left\{J(S): S \supseteq \Gamma, P \in \mathcal{K}_{\sigma}\right\} .
$$

Thus,

$$
\begin{aligned}
& J(\Gamma)-\frac{\epsilon}{2} \leq J(P), \\
& J(\Gamma)+\frac{\epsilon}{2} \geq J(S)
\end{aligned}
$$

for some $S \in \mathcal{K}_{\sigma}$ and some $P \in \mathcal{K}_{\delta}$ with $P \subseteq \Gamma \subseteq S$. Putting these inequalities together we obtain

$$
J(S)-\frac{\epsilon}{2} \leq J(\Gamma) \leq J(P)+\frac{\epsilon}{2}
$$

and therefore

$$
J(S)-\epsilon \leq J(P) .
$$

We know that $J(P)=L(\mu)(X)-\delta(P)$ and $J(S)=L(\mu)(X)-\delta(S)$, where $\delta$ is the measure defect function. On the other hand, $\Gamma$ satisfies Hall's condition and therefore $S \supseteq \Gamma$ satisfies Hall's condition as well. Therefore, $\delta(S)=0$. We have $L(\mu)(X)-\epsilon \leq L(\mu)(X)-\delta(P)$, and thus, $\delta(P) \leq \epsilon$.

The following proposition gives an example of types of algebras $\mathcal{K}$ to which Proposition 2.3 can be applied.

Proposition 2.4. Let $X$ and $Y$ be Loeb measurable sets of finite measure. Let $\mathcal{K}$ be an internal algebra of internal graphs in $X \times Y$ such that

$$
J(\Delta)=L(\mu)(\operatorname{dom}(\Delta))
$$

for every $\Delta \in \mathcal{K}$. Then, $J$ is strongly subadditive. Consequently, every Souslin graph over $\mathcal{K}$ satisfying Hall's condition possesses matchings of arbitrary small measure defects.

Proof. Let $\Gamma, \Delta \in \mathcal{K}$. Note that always have $\operatorname{dom}(\Gamma \cap \Delta) \subseteq \operatorname{dom}(\Gamma) \cap \operatorname{dom}(\Delta)$ and $\operatorname{dom}(\Gamma \cup \Delta)=\operatorname{dom}(\Gamma) \cup \operatorname{dom}(\Delta)$. Therefore, $J(\Gamma \cup \Delta)+J(\Gamma \cap \Delta)=$ $L(\mu)(\operatorname{dom}(\Gamma \cup \Delta))+L(\mu)(\operatorname{dom}(\Gamma \cap \Delta)) \leq L(\mu)(\operatorname{dom}(\Gamma) \cup \operatorname{dom}(\Delta))+$ $L(\mu)(\operatorname{dom}(\Gamma) \cap \operatorname{dom}(\Delta))=L(\mu)(\operatorname{dom}(\Gamma))+L(\mu)(\operatorname{dom}(\Delta))=J(\Gamma)+J(\Delta)$.

Now, we give an example of a situation where the condition of Proposition 2.4. is satisfied. We start with an internal set $\mathcal{I}$ of internal injections the graphs of which are the subsets of the product of two Loeb measurable sets $X$ and $Y$ of finite measure. We suppose that every two functions $\varphi$ and $\psi$ in $\mathcal{I}$ coincide on their common range. That is, if $\varphi(x)$ is in the range of $\psi$ then $x$ must be in the domain of $\psi$ and $\varphi(x)=\psi(x)$. Also, we suppose that every internal subset of an element in $\mathcal{I}$ is an element of $\mathcal{I}$ as well. The proof of the next claim is obvious. 
Claim I. $\mathcal{I}$ is closed w.r.t. finite intersections. Therefore, the set $\mathcal{K}$ of all finite unions of elements of $M$ is an algebra of internal graphs in $X \times Y$.

Claim II. The algebra $\mathcal{K}$ satisfies the condition of Proposition 2.4. Therefore, every Souslin graph over $\mathcal{K}$ satisfying Hall's condition possesses internal matchings of arbitrary small $L(\mu)$ defects.

Proof. A typical element of the algebra $\mathcal{K}$ is a finite union of elements from $\mathcal{I}, f=$ $\varphi_{1} \cup \cdots \cup \varphi_{n}$. To prove that $J(f)=L(\mu)(\operatorname{dom}(f))$ we construct an internal matching $\varphi$ of $f$ saturating all of the domain of $f$. Let us define a sequence $A_{k},(k=1, \ldots, n)$ of internal sets as $A_{1}=\operatorname{dom}\left(\varphi_{1}\right)$ and inductively, $A_{k+1}=\operatorname{dom}\left(\varphi_{k+1}\right) \backslash \bigcup_{i=1}^{n} A_{i}$. It is clear that $A_{k},(k=1, \ldots, n)$ is a disjoint partition of $\operatorname{dom}(f)$. Now, for our matching $\varphi$ we define $\varphi=\bigcup_{k=1}^{n}\left(\varphi_{k} \mid A_{k}\right)$. The fact that the functions $\varphi_{k}$ coincide on the same range implies that $\varphi$ is indeed an injection.

We conclude this section with another interesting sufficient condition for the existence of internal matchings of a Souslin graph $\Gamma$ of arbitrary small measure defects. The condition requires that the sets in the Souslin representation of $\Gamma$ are well spread in the sense that the information about the measure defects is not lost when passing from one level of sets to the next. The crucial property used in the course of the proof is that the functional $I$ defined earlier passes trough countable, monotone unions and intersections of internal sets.

Given a sequence $s$ of integers and an integer $k$ the concatenation of $s$ and $k$ is denoted as $(s, k)$.

Proposition 2.5. Let $X$ and $Y$ be Loeb measurable of finite measure. Let $\Gamma \subseteq$ $X \times Y$ be a Souslin graph with a regular Souslin representation as

$$
\Gamma=\bigcup_{\xi \in \omega^{\omega}} \bigcap_{n \in \omega} W_{\xi \mid n} .
$$

Suppose that for every $m \in \omega$ and any sequence $s_{1}, \ldots, s_{n}$ of finitely many finite sequences of integers of length $m$ we have

$$
I\left(\bigcup_{i=1}^{m} W_{s_{i}}\right)=\sup \left\{I\left(\bigcup_{i=1}^{m} \bigcup_{k=0}^{p} W_{\left(s_{i}, k\right)}\right): p \in \omega\right\} .
$$

Suppose that Hall's condition is satisfied. Then, for every $\epsilon>0, \Gamma$ has an internal matching of measure defect $\leq \epsilon$.

Proof. We construct a particular sequence $\xi_{0} \in \omega^{\omega}$ as follows. We have $\Gamma \subseteq$ $\bigcup_{n \in \omega} W_{n}$. Therefore, $\bigcup_{n \in \omega} W_{n}$ satisfies Hall's condition and its measure defect is 0 . Consequently, there exists $n_{0} \in \omega$ with $I\left(\bigcup_{i=0}^{n_{0}} W_{i}\right)>L(\mu)(X)-\epsilon$. Define $\xi_{0}(0)=$ $n_{0}$. Now, by the condition above there exists $n_{1} \in \omega$ with $I\left(\bigcup_{i=0}^{n_{0}} \bigcup_{k=1}^{n_{1}} W_{(i, k)}\right)>$ $L(\mu)(X)-\epsilon$. Define $\xi_{0}(1)=n_{1}$. Continuing inductively we construct a sequence $\xi_{0}$ such that for every $n \in \omega$,

$$
I\left(\bigcup\left\{W_{s}: s \in \omega^{n}, s \leq \xi_{0}\right\}\right)>L(\mu)-\epsilon,
$$

where $s \leq \xi_{0}$ means $s(i) \leq \xi_{0}(i)$ for every $i$ in the common domain of $s$ and $\xi_{0}$. It is well known (see [BrSi], Lemma 4.6) that

$$
\bigcup_{\xi \leq \xi_{0}} \bigcap_{n \in \omega} W_{\xi \mid n}=\bigcap_{n \in \omega} \bigcup\left\{W_{s}: s \in \omega^{n}, s \leq \xi_{0}\right\} .
$$


Let $\Gamma_{0}$ be the graph on the right hand side above. $\Gamma_{0}$ is $\Pi_{1}^{0}$ and $\Gamma_{0} \subseteq \Gamma$. The sequence $\bigcup\left\{W_{s}: s \in \omega^{n}, s \leq \xi_{0}\right\},(n \in \omega)$ is decreasing with members having the $I$ value greater than $L(\mu)(X)-\epsilon$. As the functional $I$ passes trough any monotone and decreasing sequence of internal sets we conclude that $I\left(\Gamma_{0}\right) \geq L(\mu)(X)-\epsilon$. But, for $\Pi_{1}^{0}$ graphs we have $I\left(\Gamma_{0}\right)=L(\mu)(X)-\delta\left(\Gamma_{0}\right)$ and the result follows.

\section{3. $\Pi_{2}^{0}$ AND $\kappa$-SOUSLIN GRAPHS}

In this section we consider $\Pi_{2}^{0}$ and $\kappa$-Souslin graphs. We first give a necessary and sufficient condition for the existence of an internal matching of the measure defect $\leq \epsilon$ for $\Pi_{2}^{0}$ graphs. Then, we define $\Pi_{2}^{0}(\kappa)$ graphs of depth $\omega$ and prove that Hall's condition for those types of graphs implies the existence of internal matchings of arbitrary small measure defects provided that the cofinality of $\kappa$ is $>\omega$. At the end a similar proposition for $\kappa$-Souslin graphs is given.

Proposition 3.1. Let $X$ and $Y$ be Loeb measurable sets of finite measure and let $\Gamma \subseteq X \times Y$ be a $\Pi_{2}^{0}$ graph

$$
\Gamma=\bigcap_{m \in \omega} \bigcup_{n \in \omega} W_{m, n}
$$

Then, $\Gamma$ has an internal matching of measure defect $\leq \epsilon$ if and only if there exists a sequence $n_{k},(k \in \omega)$ of natural numbers such that every finite intersection of elements of the sequence

$$
\bigcup_{i=1}^{n_{k}} W_{k, i}
$$

has measure defect $\leq \epsilon$.

Proof. Let $\mathcal{M}_{\epsilon} \subseteq \Gamma=\bigcap_{m \in \omega} \bigcup_{n \in \omega} W_{m, n}$ be an internal matching of measure defect $\leq \epsilon$, i.e., $\delta\left(\mathcal{M}_{\epsilon}\right) \leq \epsilon$. Then, by $\omega_{1}$ saturation, for every $k \in \omega$ there exists an integer $n_{k}$ with $\mathcal{M}_{\epsilon} \subseteq \bigcup_{i=1}^{n_{k}} W_{k, i}$. Moreover, every finite intersection of sets of the sequence $\bigcup_{i=1}^{n_{k}} W_{k, i},(k=1,2, \ldots)$ contains $\mathcal{M}_{\epsilon}$ and, thus, has the measure defect $\leq \epsilon$.

Conversely, let a sequence $n_{k}$ fulfill (1). Then $\bigcap_{k \in \omega} \bigcup_{i=1}^{n_{k}} W_{k, i} \subseteq \Gamma$ is a $\Pi_{1}^{0}$ set of the measure defect $\leq \epsilon$ because, as mentioned earlier, the functional $I$ from $\S 2$ passes trough the decreasing and monotone intersections of internal graphs.

A $\Pi_{2}^{0}(\kappa)$ graph of depth $\omega$ is a graph of the form

$$
\bigcap_{n \in \omega} \bigcup_{\alpha \in \kappa} W_{\alpha}^{n}
$$

where $W_{\alpha}^{n},(n \in \omega, \alpha<\kappa)$ are internal graphs satisfying $W_{\alpha}^{n} \subseteq W_{\beta}^{n}$ for $\alpha<\beta$ and every $n \in \omega$. One should stress here that it is not true that an arbitrary $\Pi_{2}^{0}$ graph is at the same time $\Pi_{2}^{0}(\kappa)$ graphs of depth $\omega$, unless the cofinality of $\kappa$ is $\omega$.

Proposition 3.2 ( $\kappa^{+}$saturation). Suppose that the cofinality of $\kappa$ is at least $\omega_{1}$. Let $X$ and $Y$ be Loeb measurable sets of finite measure and let $\Gamma \subseteq X \times Y$ be $a \Pi_{2}^{0}(\kappa)$ graph of depth $\omega$. Suppose that Hall's condition $\left(\mathcal{H}_{e}\right)$ is satisfied, i.e., 
$L(\mu)_{e}(\Gamma(A)) \geq L(\mu)(A)$ for every internal $A \subseteq X$. Then, $\Gamma$ has internal matchings of arbitrary small measure defects.

Proof. Let us first note that we may suppose that

$$
\bigcup_{\alpha \in \kappa} W_{\alpha}^{m} \subseteq \bigcup_{\alpha \in \kappa} W_{\alpha}^{n}
$$

for every fixed $m$ and $n$ with $m>n$. Indeed, we have $\left(\bigcup_{\alpha \in \kappa} W_{\alpha}^{m}\right) \cap\left(\bigcup_{\beta \in \kappa} W_{\alpha}^{n}\right)=$ $\bigcup_{\alpha, \beta}\left(W_{\alpha}^{m} \cap W_{\beta}^{n}\right)=\bigcup_{\alpha \in \kappa}\left(W_{\alpha}^{m} \cap W_{\alpha}^{n}\right)$ and the family $W_{\alpha}^{m} \cap W_{\alpha}^{n}$ increases as $\alpha$ increases. Therefore, $\bigcup_{\alpha \in \kappa} W_{\alpha}^{n}$ may be replaced by $\bigcap_{k=1}^{n} \bigcup_{\alpha \in \kappa} W_{\alpha}^{k}$ if necessary.

Let us call the sets $W_{\alpha}^{m},(\alpha<\kappa)$ the sets of rank $m$. Using the fact that $\Gamma \subseteq$ $\bigcup_{\alpha \in \kappa} W_{\alpha}^{1}$ we have that Hall's condition is satisfied for $\bigcup_{\alpha \in \kappa} W_{\alpha}^{1}$. The later graph is a monotone union of $\kappa$ many internal sets and therefore, given $\epsilon>0$, there exists a set $W_{\alpha_{1}^{1}}$ of the first level such that

$$
\delta\left(W_{\alpha_{1}^{1}}\right)<\epsilon
$$

The same is true for the sets of the second (and any other) level. So, there exists a set $W_{\alpha_{2}^{1}}$ of the second level and a set $W_{\alpha_{1}^{2}}$ of the first level, such that $\delta\left(W_{\alpha_{1}^{2}}\right)<\epsilon$ and such that the following diagram holds

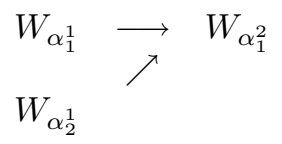

where the notation $A \longrightarrow B$ means that $A$ is a subset of $B$. Namely, for $W_{\alpha_{2}^{1}}$ we take a set of the second level such that $\delta\left(W_{\alpha_{2}^{1}}\right)<\epsilon$. Using the fact $W_{\alpha_{2}^{1}} \subseteq \bigcup_{\alpha \in \kappa} W_{\alpha}^{1}$ the $\kappa$ saturation assumption insures the existence of a set $W_{\alpha_{1}^{2}}$ such that the above diagram exists.

In general, we may construct the following diagram

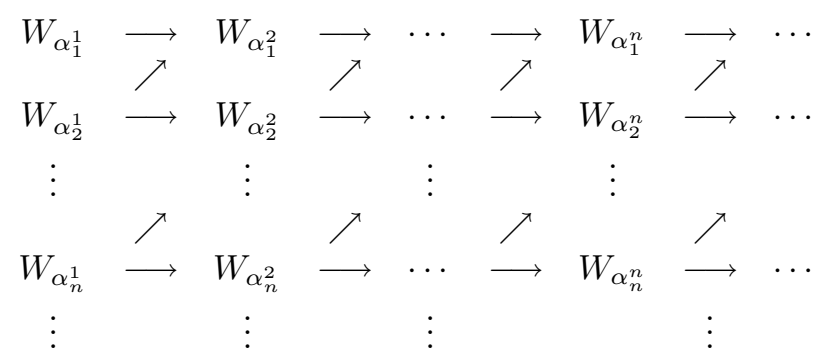

That is, we may construct a sequence $W_{\alpha_{m}^{n}}$ of sets of $n$-th level such that

$$
\begin{gathered}
W_{\alpha_{m}^{n}} \subseteq W_{\alpha_{m}^{n+1}}, \\
W_{\alpha_{n}^{1}} \subseteq W_{\alpha_{n-1}}^{2} \subseteq \cdots \subseteq W_{\alpha_{1}^{n}}, \\
\delta\left(W_{\alpha_{m}^{n}}\right)<\epsilon,
\end{gathered}
$$

for every $m, n$. But, the cofinality of the cardinal $\kappa$ is $>\omega$, so there exists a sequence $W_{m}$ of graphs such that $W_{m}$ is of level $m$ and $W_{\alpha_{m}^{i}} \subseteq W_{m}$ for all $i$. The intersection 
$\bigcap_{m \in \omega} W_{m}$ is a subset of $\Gamma$ and we show that every finite intersection of sets $W_{m}$ has $L(\mu)$ defect $<\epsilon$ which will imply that $\Gamma$ has an internal matching of measure defect $<\epsilon$. Indeed, for every $m$ and $n$ we have $W_{m} \supseteq W_{\alpha_{m}^{n}} \supseteq W_{\alpha_{m+n}^{1}}$ and in particular $W_{k} \supseteq W_{\alpha_{k}^{m-k}} \supseteq W_{\alpha_{m}^{1}}$. So, $\bigcap_{k=1}^{m} W_{k} \supseteq W_{\alpha_{m}^{1}}$ and the claim is proved because $\delta\left(W_{\alpha_{m}^{1}}\right)<\epsilon$.

In the same manner as above we can prove the next proposition. A $\kappa$-Souslin graph is a graph of the form

$$
\bigcup_{\xi \in \kappa^{\omega}} \bigcap_{n \in \omega} W_{\xi \mid n}
$$

where $W_{s},\left(s \in \kappa^{\omega}\right)$ is a system of internal graphs. If $W_{s} \subseteq W_{t}$ for $t \supseteq s$ we call it regular.

Proposition 3.3 ( $\kappa^{+}$saturation). Let $\kappa$ be, as before, a cardinal of cofinality $\geq \omega_{1}$, and $X$ and $Y$ Loeb measurable sets of finite measure. Let $\Gamma \subseteq X \times Y$ be a regular $\kappa$-Souslin graph as above. Suppose that $W_{s} \subseteq W_{t}$ whenever $s$ and $t$ are sequences of the same length satisfying $s(n) \leq t(n)$ for every $n$ belonging to the domain of $s$ (and thus $t$ ). Suppose that Hall's condition $\mathcal{H}_{e}$ is satisfied. Then, $\Gamma$ has internal matchings of arbitrary small measure defects.

\section{Graphs possessing internal, $\Pi_{1}^{0}$, OR $\Sigma_{1}^{0}$ Liftings}

A lifting in Nonstandard Analysis is usually meant to be an internal object, e.g. a function, approximating a standard object almost surely. For example, a lifting of a real valued, measurable function $f$ defined on a bounded Loeb space $M$ is an internal function $\varphi$ taking the values in ${ }^{*} R$ such that $f(x)$ and $\varphi(x)$ are infinitely close for almost all $x$. Lead by a similar idea we say that a graph $\Gamma$ possesses an internal $\left(\Pi_{1}^{0}\right.$ or $\left.\Sigma_{1}^{0}\right)$ lifting if it can be approximated by an internal $\left(\Pi_{1}^{0}\right.$ or $\left.\Sigma_{1}^{0}\right)$ graph $\Delta$ such that almost all of the $Y$ sections of $\Delta$ and $\Gamma$ are identical. More formally we give the following

Definition 4.1. Let $\Gamma$ and $\Delta$ be graphs in $X \times Y$, with $X$ and $Y$ of finite Loeb measure. We say that $\Delta$ is a lifting of $\Gamma$ if for almost all $x \in \operatorname{dom}(\Gamma) \cup \operatorname{dom}(\Delta)$, $\Gamma(x)=\Delta(x)$. If $\Delta$ is $\Pi_{1}^{0}\left(\Sigma_{1}^{0}\right.$ or internal $)$ then $\Delta$ is called a $\Pi_{1}^{0}\left(\Sigma_{1}^{0}\right.$, internal $)$ lifting of $\Gamma$.

The importance of liftings for the version of Hall's theorem that we are dealing with in this paper lies in a simple fact that if $\Delta$ is a lifting of $\Gamma$ and if $\Gamma$ satisfies Hall's condition then $\Delta$ satisfies Hall's condition as well. This transforms the question of the validity of Hall's theorem for $\Gamma$ to the question of the existence of internal $\Pi_{1}^{0}$ or $\Sigma_{1}^{0}$ liftings of $\Gamma$.

Lemma 4.2. Let $X$ and $Y$ be Loeb measurable sets of finite measure and let $\Gamma \subseteq X \times Y$ be arbitrary graph such that Hall's condition $\mathcal{H}_{i}$ holds for $\Gamma$, i.e., $L(\mu)_{i}(\Gamma(A)) \geq L(\mu)(A)$ for every internal $A \subseteq X$. Suppose that $\Delta \subseteq X \times Y$ a lifting of $\Gamma$. Then, Hall's condition $\mathcal{H}_{i}$ holds for $\Delta$ as well.

Proof. It easy to see that if Hall's condition $\mathcal{H}_{i}$ holds then the formulation of $\mathcal{H}_{i}$ in which the internal sets $A$ are replaced by arbitrary Loeb measurable sets $M$ also holds (see Lemma 2.1 in [Ži1]). As $\Delta$ is a lifting of $\Gamma$ we have $\Gamma(x)=\Delta(x)$ for almost all $x \in X$. Let $A \subseteq X$ be internal and let $M=\{x \in A: \Gamma(x)=\Delta(x)\}$. 
Then, $M$ is Loeb measurable and $L(\mu)(M)=L(\mu)(A)$. We have $\Gamma(M)=\Delta(A)$ so $L(\mu)_{i}(\Delta(A))=L(\mu)_{i}(\Gamma(M)) \geq L(\mu)(M)=L(\mu)(A)$ and we are done.

The existence of internal, $\Pi_{1}^{0}$, and $\Sigma_{1}^{0}$ liftings for different types of graphs has been extensively investigated so far in a series of papers ([Ži3], [Ži4] and [Ži5]). The complete solution in the case of the vertical sections being of the first two levels of Borel hierarchy is provided in [Ži5], Theorem 1, e) and Corollary 5, c). The largest class of graphs possessing $\Pi_{1}^{0}$ liftings is the class of $\Sigma_{1}^{1}$ graphs with $\Pi_{1}^{0} Y$-sections. The largest class of graphs possessing $\Sigma_{1}^{0}$ liftings is the class of $\Pi_{1}^{1}$ graphs with $\Sigma_{1}^{0}$, $Y$-sections. Finally, the largest class of graphs possessing internal liftings is the class of $\Pi_{1}^{1}$ graphs with internal $Y$-sections.

The complete answer for arbitrary Borel graphs is given in [SchŽi]. Every Borel graph $\Gamma$ all of whose vertical sections are $\Sigma_{\alpha}^{0}\left(\Pi_{\alpha}^{0}\right)$ possesses a $\Sigma_{\alpha}^{0}\left(\Pi_{\alpha}^{0}\right)$ lifting.

Notice that if a graph possesses a $\Pi_{1}^{0}$ or $\Sigma_{1}^{0}$ lifting then it must have a measurable domain. Therefore the above results are the best possible. For example, it is not true that a $\Pi_{1}^{1}$ graph all of whose $Y$-sections are $\Pi_{1}^{0}$ sets possesses a $\Pi_{1}^{0}$ lifting. Let $f$ be a graphs of a $\Pi_{1}^{1}$ function in the unit square $[0,1] \times[0,1]$ whose domain is not Lebesgue measurable (take a $\Pi_{1}^{1}$ uniformization of a $\Pi_{1}^{1}$ subset of $[0,1] \times[0,1]$, the domain of which is a nonmeasurable $\Sigma_{2}^{1}$ subset of $\left.[0,1]\right)$. Let $\Gamma$ be the standard part inverse image of the graph of $f$. Then, $\Gamma$ is a $\Pi_{1}^{1}$ graph all of whose vertical sections are $\Pi_{1}^{0}$ sets and with the nonmeasurable domain. Therefore, $\Gamma$ does not possess a $\Pi_{1}^{0}$ lifting.

We summarize all the above in the following

Proposition 4.3. Let $X$ and $Y$ be Loeb measurable sets of finite measure. Let $\Gamma \subseteq X \times Y$ be a graph. Then the following is true.

a) If $\Gamma$ is a $\Sigma_{1}^{1}$ graph all of whose $Y$ sections are $\Pi_{1}^{0}$ sets or a $\Pi_{1}^{1}$ graphs all of whose $Y$ sections are internal sets, and if the inner Hall's condition holds, then $\Gamma$ possesses a $\Sigma_{1}^{0}$ matching saturating almost all of $X$.

b) If $\Gamma$ is a $\Pi_{1}^{1}$ graph all of whose $Y$ sections are $\Sigma_{1}^{0}$ sets and if the inner Hall's condition holds then $\Gamma$ possesses internal matchings of arbitrary small measure defects.

\section{Graphs of Finite $L(\nu)$ Measure}

As we have seen so far, it is generally difficult to claim the existence of an internal matching of a certain $L(\mu)$ defect of a (say, Loeb measurable) graph $\Gamma$ satisfying Hall's condition. One of the reasons for this lies in the fact that, when we approximate a given graph by, for instance, an internal graph (in the sense of product measure), we cannot claim that i) the new graph satisfies Hall's condition and, ii) that a matching for a new graph would be a matching for the old one as well. Although the first problem can be easily solved by choosing some suitable approximation (for example, approximating by a $\Pi_{1}^{0}$ graph from above) it seems that the second obstacle cannot be surpassed so easily. A matching of the approximating graph does not have to be related to the original graph whatsoever. The reason for that lies in the fact that the $L(\mu \times \mu)$ measure of an internal matching $\mathcal{M}$ is always equal to zero. A solution to this problem that we suggest here is to change the measure $L(\mu \times \mu)$ in the product space $X \times Y$ to a more suitable one. The new measure $L(\nu)$ will assign finite and nonvanishing measures to internal functions in $X \times Y$. Therefore the deficiency ii) will be removed for the graphs of finite $L(\nu)$ 
measure. Thus, surprisingly, Hall's theorem will hold true if either the graph in question is "fat", i.e., of full $L(\mu \times \mu)$ measure in the product, or if it is "thin", i.e., of finite $L(\nu)$ measure (and it satisfies Hall's condition).

A new internal counting measure $\nu$ in the product $\Omega \times \Omega$ is introduced by defining $\nu(W)=|W| / K$ where $W \subseteq \Omega \times \Omega$ is an internal set and $K$ an infinite integer satisfying $K / H \approx 1$. In such a way we obtain an internal measure space $(\Omega \times$ $\Omega, \Sigma \times \Sigma, \nu)$. The corresponding Loeb measure of $\nu$ is, as usual, denoted by $L(\nu)$. Note that for every internal $A \subseteq \Omega$ and $W \subseteq \Omega \times \Omega$ with $|A|=|W|$, we have $L(\mu)(A)=L(\nu)(W)$. It turns out that the graphs that have finite $L(\nu)$ measure and satisfy Hall's condition almost surely contain an internal matching saturating almost all of the domain of the graph.

Proposition 5.1. Let $X$ and $Y$ be $L(\mu)$ measurable sets of finite and positive measure and let $\Gamma \subseteq X \times Y$ be a $L(\nu)$ measurable graph of finite $L(\nu)$ measure. Then, the following three conditions are equivalent.

i) There exists an internal matching $\mathcal{M}$ saturating almost all elements of $X$ and such that

$$
L(\mu)(\{x:(x, \mathcal{M}(x)) \notin \Gamma\})=0 .
$$

ii) The outer Hall's condition holds, i.e.,

$$
L(\mu)_{e}(\Gamma(A)) \geq L(\mu)(A)
$$

for every internal $A \subseteq X$.

iii) The inner Hall's condition holds, i.e.,

$$
L(\mu)_{i}(\Gamma(A)) \geq L(\mu)(A)
$$

for every internal $A \subseteq X$.

Proof. i) $\Rightarrow$ iii). Let $M=\{x:(x, \mathcal{M}(x)) \notin \Gamma\}$. By our assumption we know that $L(\mu)(M)=0$. Now, we have $L(\mu)_{i}(\Gamma(A)) \geq L(\mu)_{i}(\Gamma(A \backslash M)) \geq L(\mu)_{i}(\mathcal{M}(A \backslash M))=$ $L(\mu)(A \backslash M)=L(\mu)(A)$, where we used an obvious fact that internal 1-1 functions preserve counting measures.

iii) $\Rightarrow$ ii) is obvious.

ii) $\Rightarrow$ i). Using the fact that the $L(\nu)$ measure of $\Gamma$ is finite there exists a $\Pi_{1}^{0}$ graph $\Delta \supseteq \Gamma$ in $X \times Y$ with $L(\nu)(\Delta / \Gamma)=0$. As $\Delta$ contains $\Gamma$ it satisfies Hall's condition. Consequently, $\Delta$ has a $\Sigma_{1}^{0}$ matching $\mathcal{M}$ saturating almost all $X$ (Proposition 1.2 in [Ži 1$]$ ). Moreover, $L(\nu)(\mathcal{M})=L(\mu)(X)>0$ and therefore, $L(\mu)(\{x:(x, \mathcal{M}(x)) \notin \Gamma\})=0$.

Corollary 5.2. Let $X$ and $Y$ be $L(\mu)$ measurable sets of finite measure and let $\Gamma \subseteq X \times Y$ be $a \Sigma_{1}^{1}$ or a $\Pi_{1}^{1}$ graph of finite $L(\nu)$ measure. Let the inner Hall's condition be satisfied. Then, $\Gamma$ possesses a $\Sigma_{1}^{0}$ matching saturating almost all of $X$.

Proof. First note that every $\Sigma_{1}^{1}$ or $\Pi_{1}^{1}$ graph is $L(\nu)$ measurable. By the previous proposition there exists an internal injective function $\mathcal{M}$ such that $L(\mu)(\{x:(x, \mathcal{M}(x)) \notin \Gamma\})=0$. Let $\mathcal{N}=\mathcal{M} \cap \Gamma$. Then, $\mathcal{N}$ is a $\Sigma_{1}^{1}$ or a $\Pi_{1}^{1}$ matching of $\Gamma$ saturating almost all of $X$. The result now follows from the fact that any $\Sigma_{1}^{1}$ or any $\Pi_{1}^{1}$ function is a.e. equal to a $\Sigma_{1}^{0}$ sub-function $f$. 
One could hope, at a first sight, that Proposition 5.1 and Corollary 5.2 would also work if one releases the requirement that $\Gamma$ is of finite $L(\nu)$ measure and requires only that $\Gamma$ is $L(\nu)$ measurable (meaning that $\Gamma \cap F$ is measurable for every set $F$ of finite $L(\mu)$ measure). We might try to prove then that for any unbounded Loeb measure $L(\nu)$ and any Loeb measurable set $M$ (of finite or infinite measure) there exists a $\Pi_{1}^{0}$ set $P \supseteq M$ so that $L(\mu)(P / M)=0$. However, this would imply that any Borel graph satisfying Hall's condition, for example, would possesses a $\Sigma_{1}^{0}$ matching saturating almost all of $X$, which is impossible. In particular, the $\Sigma_{1}^{0}$ graph $\Gamma$ in the example on page 396 in [Ži1] cannot be approximated from outside by a $\Pi_{1}^{0}$ graph in the above manner.

Concerning the general approximating question on unbounded Loeb measurable spaces raised above, it has been further proved in [Ži6] (answering a question posed at the Galaxy news group) that for any nontrivial (i.e., non Borel) $\Pi_{1}^{1}$ set $D$, there exists an unbounded counting Loeb measure $L\left(\mu_{D}\right)$ so that $D$ is not a.e. equal to any Borel set $B$. Further, given $L\left(\mu_{D}\right)$, there exists a $\Pi_{2}^{0}$ set $R$ which cannot be approximated from within or from outside by a $\Sigma_{1}^{0}$ or a $\Pi_{1}^{0}$ set.

\section{REFERENCES}

[Ar] Z. Artstein, Distributions of Random Sets and Random Selections, Israel Journal of Math. 46 (1983), 313-324. MR 85m:60017

[Be] E. Bertossi, Standard Capacities With Nonstandard Pavings, Notas De LA Sociedad De Mat. De Chile V (1986), 1-6. MR 88c:26023

[BoVa] B. Bollobas and N. Th. Varopoulos, Representation of Systems of Measurable Sets, Math.Proc.Camb.Phil.Soc. 78 (1974), 323-325. MR 52:686

[BrSi] D. W. Bressler and M. Sion, The Current Theory of Analytic Sets, Can.J.Math. 16 (1964), 207-230. MR 29:1153

[Ha] P. Hall, On Representatives of Subsets, J. London Math. Soc. 10 (1935), 26-30.

[He] C. W. Henson, Analytic Sets, Baire Sets, and the Standard Part Map, Canadian J. Math. 31 (1979), 663-672. MR 80i:28019

[HeRo] C. W. Henson and D. Ross, Analytic mappings on hyperfinite sets, Proc.Amer.Math.Soc. 2 (1993), 587-596. MR 93g:03055

[HuLo] A. E. Hurd and P. A. Loeb, An Introduction to Nonstandard Real Analysis, Academic Press, New York, 1985. MR 87d:03184

[KKLM] H. J. Keisler, K. Kunen, A. Miller and S. Leth, Descriptive Set Theory over Hyperfinite sets, J. Symbolic Logic 54 (4) (1989), 1167-1180. MR 91c:03040

[Lo] P. A. Loeb, Conversion from Nonstandard to Standard Measure Space and Applications in Probability Theory, Trans.Amer.Math.Soc. 211 (1975), 113-122. MR 52:10980

[Ma] Mauldin, D., One-to-one Selections-marriage Theorems, American Journal of Mathematics 104 (1982), 823-828. MR 84b:28013

[Me] P. A. Meyer, Probabilités et Potentiel, Hermann, Paris, 1966. MR 34:5118

[NW] C. Nash-Williams, Unexplored and Semi-Explored Territories in Graph Theory, New Directions in Graph Theory (Frank Harray, ed.), Academic Press, 1973. MR 52:7944

[Ro] D. Ross, Lifting Theorems in Nonstandard Measure Theory, Proc.Amer.Math.Soc 109 (1990), 809-822. MR 91b:03110

[SchŽi] K. Schilling and B. Živaljević, Louveau's Theorem for the Descriptive Set Theory of Internal Sets, (submitted).

[StBa] K. D. Stroyan, J. M. Bayod, Foundations of Infinitesimal Stochastic Analysis, NorthHolland, Amsterdam, 1986. MR 87m:60001

[Ži1] B. Živaljević, Hyperfinite Transversal Theory, Trans. Amer. Math. Soc 330 (1992), 371399. MR 92f:03073

[Ži2] _ Rado's Theorem for the Loeb Space of an internal *-finitely additive measure space, Proc. Amer. Math. Soc. 112 (1991), 203-207. MR 91h:03092

[Ži3] , The structure of graphs all of whose $Y$ sections are internal sets, J. of Symbolic Logic 56 (1991), 50-66. MR 92j:03059 
[Ži4] _ Graphs with $\Pi_{1}^{0}(\kappa) Y$ sections, Arch. Math. Logic 32 No.4 (1993), 259-273. MR 94e:03065

[Ži5] —,$\Pi_{1}^{1}$ functions are almost internal, Trans. Amer. Math. Soc. 347 (1995), 26212632.

[Ži6] _,$\Pi_{1}^{1}$ sets of infinite Loeb measure, Proc. Amer. Math. Soc. (to appear).

Department of Computer Science, The University of Illinois at Urbana - Champaign, URBANA, IllinoIS 61801

Current address: International Paper Company, Process Management Computer, 3101 International Drive East, Mobile, Alabama 36606

E-mail address: zivaljev@cs.uiuc.edu 